\title{
Using a gyro as a tool for continuously variable lateral stabilisation of dynamic bipeds
}

doi:10.1533/abbi.2006.0032

\author{
N. M. Mayer ${ }^{1,2}$, K. Masui ${ }^{2}$, M. Browne ${ }^{3}$, M. Asada1,2 \\ and M. Ogino $0^{1,2}$ \\ ${ }^{1}$ Asada S.I. ERATO $7 S T$, Osaka, Japan \\ ${ }^{2}$ Dept. of Adaptive Machine Systems, Graduate School of Engineering, Osaka University, Osaka, Japan \\ ${ }^{3}$ CSIRO Mathematical and Information Sciences, Brisbane, Australia
}

\begin{abstract}
We present simulations of different types of biped walking machines that are stabilized in the lateral direction by adding a gyro. The simulations show that, in principle, it is indeed possible to stabilise biped walking machines. Examples presented in this work include a three-dimensional version of the simplest walking model, which is based on earlier investigations, and an actuated biped robot. Here, the gyro is used as a reaction wheel. Finally, a rotor-brake combination is outlined that was used in an artistic biped robot at the Aichi World Exhibition.
\end{abstract}

Key words: Biped, simulation, gyro.

\section{INTRODUCTION}

Walking with two legs is still one of the biggest challenges for research in humanoid robots. This is mainly due to problems that are related to balancing. Classical approaches use trajectory-based control and/or the zero moment point for statically stable walking (Sugihara and Inoue 2002).

In the last few years, approaches related to dynamic walking have steadily attracted more attention in this field. Significant examples are passive dynamic walkers (PDWs) (McGeer 1993; Collins et al. 2001). The goal of passive dynamic walking is to exploit the natural dynamics of pendulum-like legs to achieve fast and economical walking in bipedal robots. In this way, the energy consumption can be reduced significantly and the walking speed can be increased.

Motion analyses like Poincare Return Maps have been utilised in order to find the stable attractors of the physical motion dynamics, and use those for a control that is most energy efficient or optimal with respect to other eligible criteria.

Two-dimensional (2D) walkers (McGeer 1993; Hass et al. 2004; Mayer et al. 2004a, 2004b) are an intermediate step in the development of passive dynamic biped walking systems. Walkers such as those studied by McGeer and

Corresponding Author:

N. M. Mayer

Asada S.I. ERATO JST, Osaka, Japan

Tel: +81 66876 8884; Fax: +81 668768994

Email: norbert@ams.eng.osaka-u.ac.jp colleagues show a moderately stable gait at downhill without using any control whatsoever. With respect to speed, these walkers can compete with state-of-the art humanoid robots, provided the slope is sufficiently steep. The speed is, however, determined by the slope and payload, and cannot be changed for a given design.

Since the roll and yaw directions are omitted in 2D walkers, they can be seen as a kind of test unit for the stability of the dynamics of walking in the sagittal plane. Still, the development of a three-dimensional (3D) biped walker out of a 2D walker seems very difficult. It is a significant technological challenge to simultaneously control the balance in all three dimensions: pitch, roll and yaw. The starting consideration for this work was to provide a unit in which the dynamics can be changed continuously from a walker with $2 \mathrm{D}$ dynamics to a real biped by altering a single parameter that can be adjusted freely at each stage of the development. The idea here is to use a heavy fast-rotating gyro for this purpose. In this way, the rotation provides the intended stabilisation to the roll and yaw.

Gyros are symmetric rotors that are used in many technical devices like satellites, artillery, navigation units, etc. One well-known approach in robotics is the Gyrover robot (Ferreira et al. 2000), which is basically a wheel-shaped robot, rotating on its own axis, driven by an asymmetric wheel. In biped robots, several approaches have also been proposed in previous studies (Hirakoso 1996; Kuo 1999). However, the rotor was applied in both cases in a different way than in this work. In previous studies, the axis of the rotor was set parallel to the direction of motion of the robot, whereas in the present study the axis of the gyro 
is set parallel to the hip. It is well known that a gyro can have undesired effects like precession and nutation, which can disturb the walking process. Although the mathematics is well understood, it is often not amenable to numerical analysis.

For this reason, we investigated possible designs numerically. The simulations in this work have been mainly realised by using the open dynamics engine (ODE). This is an open source tool package for simulating solid body dynamics, and also includes a visualisation tool based on the open graphics library (OpenGL). Results from an investigation using a gyro to stabilise a separate, non-actuated PDW are presented in the first part of the results of this article.

In addition, the gyro can be controlled in such a way that the pitch is also controlled by the acceleration and deceleration of the rotation speed of the gyro. This feature is easy to implement in simulations. Here, the gyro or rotor serves as a reaction wheel. Thus, this reaction wheel or inertia actuator can influence the robot's movements in two ways: (1) The roll and yaw are stabilised by the rotation of the rotor; the higher the speed of the rotor, the slower the robot reacts to perturbations. (2) The pitch is stabilised by the control of the rotor acceleration, using the actio-reactio principle.

\section{GYRO-STABILISED PDW}

On the basis of these results, we investigated the behaviour of a walking model in the ODE simulation environment. Two simulation stages are described: (1) In the first stage, the aim is to reproduce the results derived for a $2 \mathrm{D}$ walker model. In the case of the kneeless walkers, we use the results from Schwab and Wisse (2001). In addition, results also exist for the 2D kneed walker (Garcia et al. 1998). Both approaches can be used as a starting point for a gyro-stabilised biped PDW. (2) In the second stage, the 2D walker is embedded in a $3 \mathrm{D}$ environment. Here, the dynamics should be the same as in the 2D approach. However, the stabilisation in the roll and yaw directions depends on the speed of the rotor. In addition, it is necessary for the walker to have a narrow hip in order to keep the forces resulting from the lateral perturbations during the single support phase as small as possible.

In the case of the kneeless walker, we investigated the $3 \mathrm{D}$ environment in conditions that are somewhat similar to conditions described by Schwab and Wisse for the simplest walking model (Figure 1). In this work, a walker of the following type is investigated. The walker has two rigid legs having length $l$, connected by a frictionless hinge at the hip. The mass is distributed over three point masses; one with mass $M$ at the hip, and two with mass $m$ at the feet. The foot mass is assumed to be negligible in comparison with the hip mass, that is, $\beta=m / M \rightarrow 0$. Hence, the motion of the walker can be described by

$$
\begin{aligned}
& \ddot{\theta}=\sin (\theta-\gamma) \text { and } \\
& \ddot{\phi}=\sin \left(\theta^{2}-\cos (\theta-\gamma)\right)+\sin (\theta-\gamma) .
\end{aligned}
$$

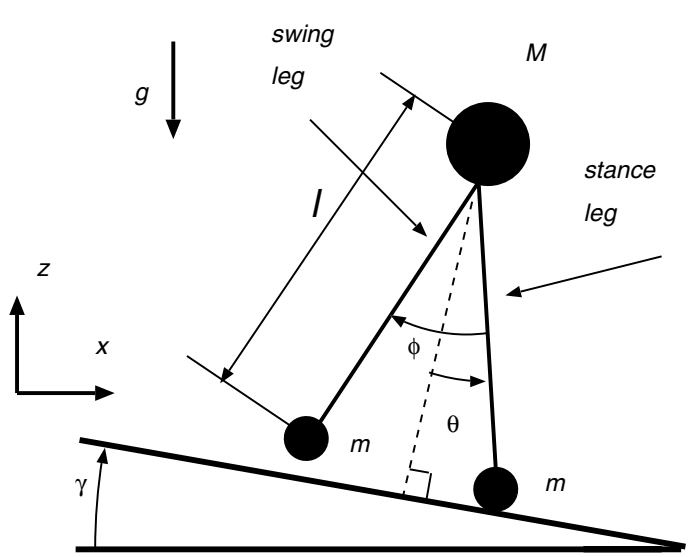

Figure 1 Simplest walking model (Schwab et al. 2001). It is a 2D model with no knees. $\theta$ indicates the angle of the stance leg to the direction of the gravitational vector and $\varphi$ the angle between both legs.

Walking with two straight legs (of same length) on a plain ground is impossible in a $2 \mathrm{D}$ model (McGeer 1990). In that approach, they used the following constraint for the heelstrike: the first time the tip of the foot can cross the line of the ground and leave the ground again. The heelstrike is then assumed to be the second time when the foot hits the ground. After the heelstrike the step is finished. The functions of stance leg and swing leg are swapped and the next step starts. The heel strike is described as an inelastic hit. The equations are as follows:

$$
\begin{aligned}
\theta_{n+1} & =-\hat{\theta}_{n}, \\
\phi_{n+1} & =-2 \hat{\theta}_{n}, \\
\dot{\theta}_{n+1} & =\cos \left(2 \hat{\theta}_{n}\right) \cdot \hat{\theta}_{n}, \quad \text { and } \\
\phi_{n+1} & =\cos \left(2 \hat{\theta}_{n}\right)\left(1-\cos \left(2 \hat{\theta}_{n}\right) \cdot \hat{\theta}_{n},\right.
\end{aligned}
$$

where $\hat{\theta}$ and $\cdot \hat{\theta}$ represent the state of $\theta$ and $\dot{\theta}$ before the heel strike. One important result of the work on the simplest walking model is that the parameters of the next step depend only on the angle of the stance $\operatorname{leg}(\theta)$ and its derivative before the previous heelstrike. In our work, we use this as an assumption. We thus reduce the search space for stable initial conditions for these two variables. As opposed to the simplest walking model, the ODE environment requires finite inertia tensors and finite dimensions of the walkers' geometries, which results in some inevitable differences between the two models. Following from this, we used the parameters as described in Table 1 for the simulated walker. The ODE simulation environment is a $3 \mathrm{D}$ environment. Since the simulation in this stage were completely free of noise, the walker could take an infinite number of steps under certain starting conditions, meaning that it was able to walk without falling to any side. Figure 2 shows the number of steps the walker was able to take as a result of the starting conditions. However, the walker remains unstable 


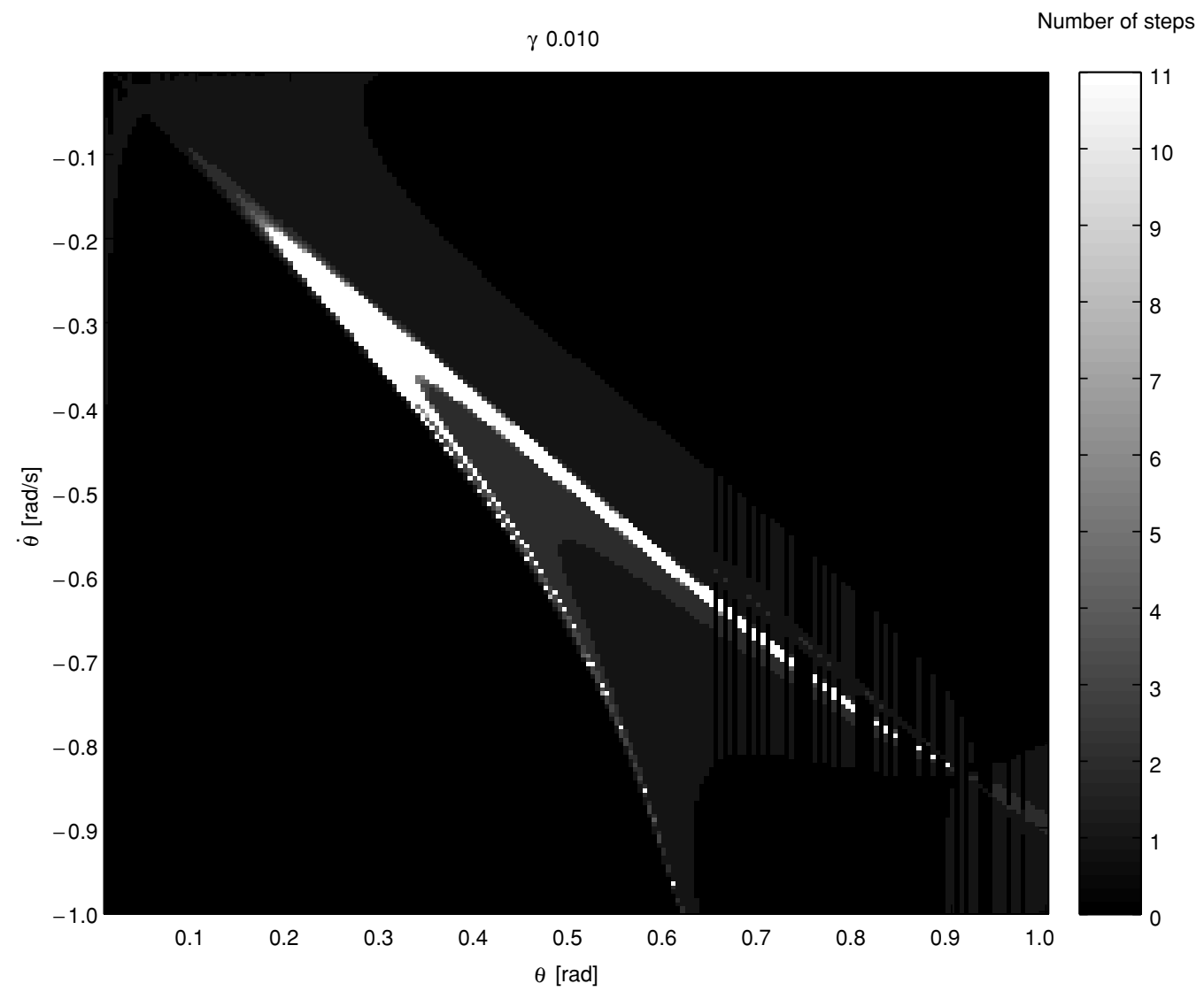

Figure 2 Stability of the kneeless walker in a noise-free 3D simulation environment. The simulation is started with a starting condition that only depends on the two parameters $\theta$ and $\dot{\theta}$. The colour of each pixel in the graph indicates how many steps the walker walks before falling. White indicates 11 steps; black, 0 steps. The simulation was stopped after 11 steps.

\begin{tabular}{lll}
\multicolumn{3}{l}{ Table 1 Parameters for the simulated walker } \\
\hline World & $g$ & $1 \mathrm{~m} / \mathrm{s}^{2}$ \\
$\quad$ Gravity & $\gamma$ & $0.01 \mathrm{rad}$ \\
Slope angle & & \\
Model & $l$ & $1 \mathrm{~m}$ \\
Leg length & $r$ & $0.04 \mathrm{~m}$ \\
Foot radius & $M_{\mathrm{H}}$ & $1 \mathrm{~kg}$ \\
Hip mass & $m$ & $0.001 \mathrm{~kg}$ \\
Leg mass & & \\
Gyro & $M_{\mathrm{G}}$ & $1 \mathrm{~kg}$ \\
Gyro mass & $I_{x}$ & $0.1 \mathrm{~kg} \mathrm{~m}^{2}$ \\
Gyro inertia & $I_{y}$ & $0.2 \mathrm{~kg} \mathrm{~m}^{2}$ \\
& $I_{z}$ & $0.1 \mathrm{~kg} \mathrm{~m}^{2}$ \\
\hline
\end{tabular}

in the roll and yaw directions. Adding lateral unsymmetrical noise to the model results in immediate destabilisation and falling of the walker. We investigated how the walking behaviour is affected by noise and how rotating the mass of the hip with a constant speed can stabilise the walker. In these simulations, the noise was simulated by random vectors in the direction parallel to the axis of the rotor, that is, perpendicular to the gravity vector and the walking direction of the motion. The results are shown in Figure 3.
For a constant level of noise, the number of steps varies, depending on the speed of the rotor. The higher the speed of the rotor, the less sensitive the device is to the noise.

\section{A PITCH BALANCE CONTROLLER}

As for the controlled pitch case, it is beneficial to fix it as closely as possible to the - possibly unstable - balance point. It is necessary for the controller (Figure 5) to have a sensor, which detects the pitch angle in relation to the controlled pitch. In the following, this angle shall be called $\alpha$. The control equation is given as follows:

$$
\dot{\omega}_{r}=A \sin \alpha+B \dot{\alpha}+C \rho(\dot{\omega}),
$$

where $\dot{\omega}_{r}$ is the control signal for the motor acceleration and $\omega$ and $\dot{\omega}$ denoting the actual speed and acceleration of the rotor, respectively. The constants $A, B$ and $C$ describe the size, weight and current shape of the robot and must be optimised as in a proportional differential (PD) controller. The angle of the pitch, $\alpha$, is detected by sensors (e.g., a gyroscope). The principle of the controller is the one of a reaction wheel. As in PD control, we have parameters that have to be determined if the values of the inertia tensors of the controlled robot and the rotor are known, which are 


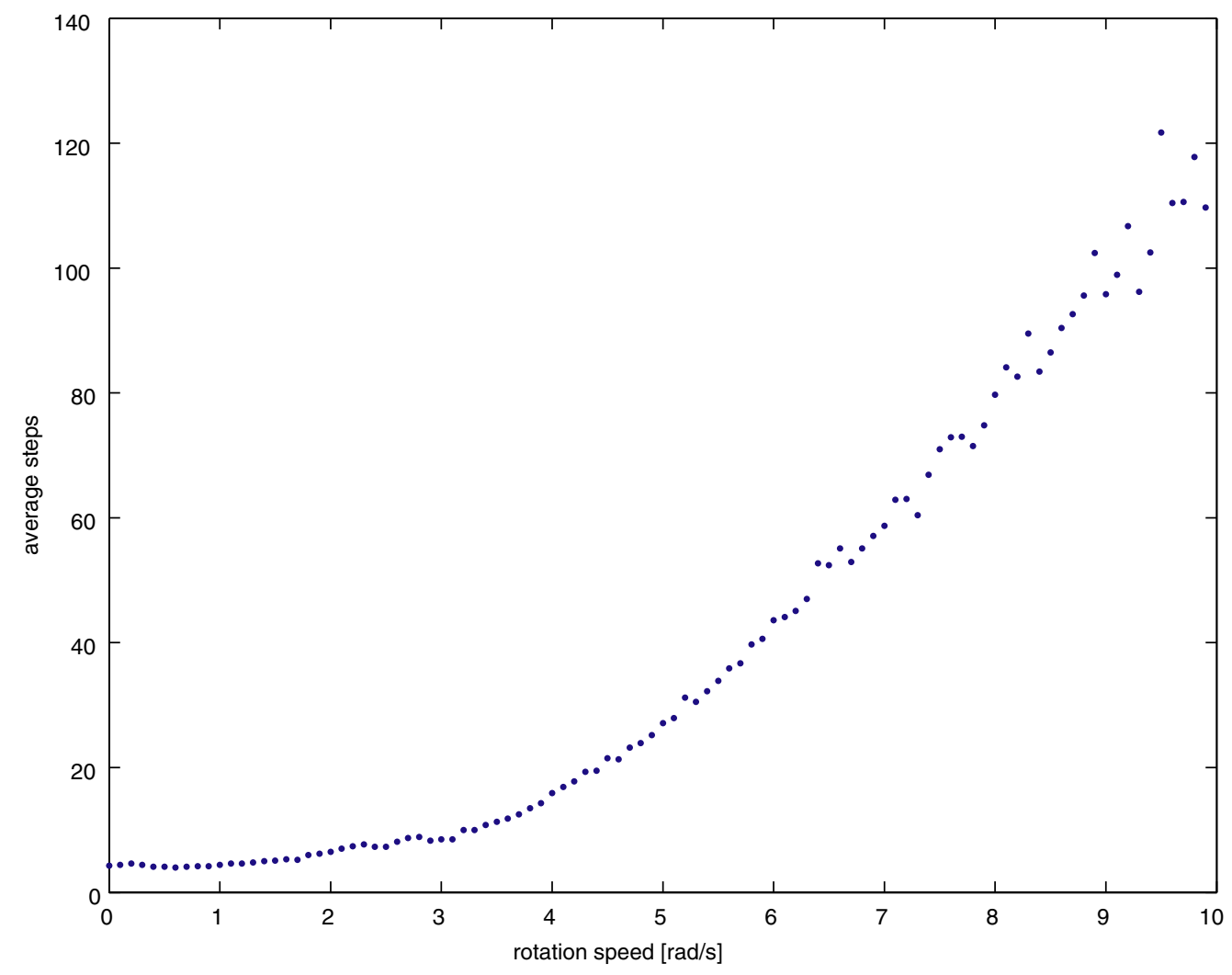

Figure 3 Stabilisation of the kneeless walker under constant noise 3D simulation. The simulation under noise shows that the number of walked steps depends on the rotation speed of the gyro. The graph shows the average number of steps (100 trials) as a function of the speed of the rotor.

comparable to the $P$ value in the PD controller paradigm. The parameter $B$ prevents the overshoot, and is thus analogous to the $D$ value of PD controller paradigm. The parameter $C$ and the function $\rho(\omega)$ can be designed to keep the speed of the rotor within an operable range. The specific design of $\rho(\omega)$ depends in particular on the type of the balance point. For example, if the balance point is unstable, the following function can be applied in the controller equation:

$$
\rho(\omega)=H\left(\omega-\omega_{\mathrm{opt}}\right),
$$

where $H(x)$ is a piecewise linear function, whose value is equal to $x$ if within the limits of $|x|<H_{\text {lim }}$ or of either $H_{\text {lim }}$ or $-H_{\lim }$ for larger or smaller values of $x$, respectively. This design makes the robot move slightly ahead of its balance point if the rotor speed is low, and lag behind if the rotor speed is too high. This causes a continuous ingression - or degression - of the rotor speed in order to balance the robot.

In case of a stable balance point, the negative value of the previous control function can be used

$$
\rho(\omega)=-H\left(\omega-\omega_{\text {opt }}\right)
$$

in order to control the rotor speed. The parameters $C$ and $H_{\text {lim }}$ should be chosen to be small enough not to interfere with the balancing, yet strong enough to keep the rotor speed within its limits and to let it converge against $\omega_{\text {opt }}$.

\section{RESULTS FOR AN ACTUATED WALKER WITH GYRO REACTION WHEEL}

The pitch balance controller was tested on a biped robot with 3 degrees of freedom in each leg. Figure 4 outlines the principle shape of the simulated robot. In the simulations, the controlled walker was able to perform the following three functions: stand up, walk and jump. For walking, the step length was taken as the variant. Standing up could be performed by a rapid acceleration of the rotor, by which the attitude of the robot could be changed. The robot was able to walk for a long period without falling down. However, the speed of the rotor tended to go out of its boundaries, so that the robot had to stop once in a while and recover the optimal speed of the rotor. The reason for this is that phases of stable and unstable balance occur during walking, and thus the optimal control (i.e., leaning forward and backward) interchanges, eventually driving the rotor speed out of its boundaries. The graph in Figure 5 shows that the walking (indicated by the regular pattern of hip movements) is anticipated by an oscillation of the pitch angle $(\alpha)$. This happened because the constant $A$ was set to relatively low value. Experiments showed that less strict pitch control resulted in better walking patterns than the more strict pitch control. In addition, the swinging leg moves downward before it strikes the ground. 


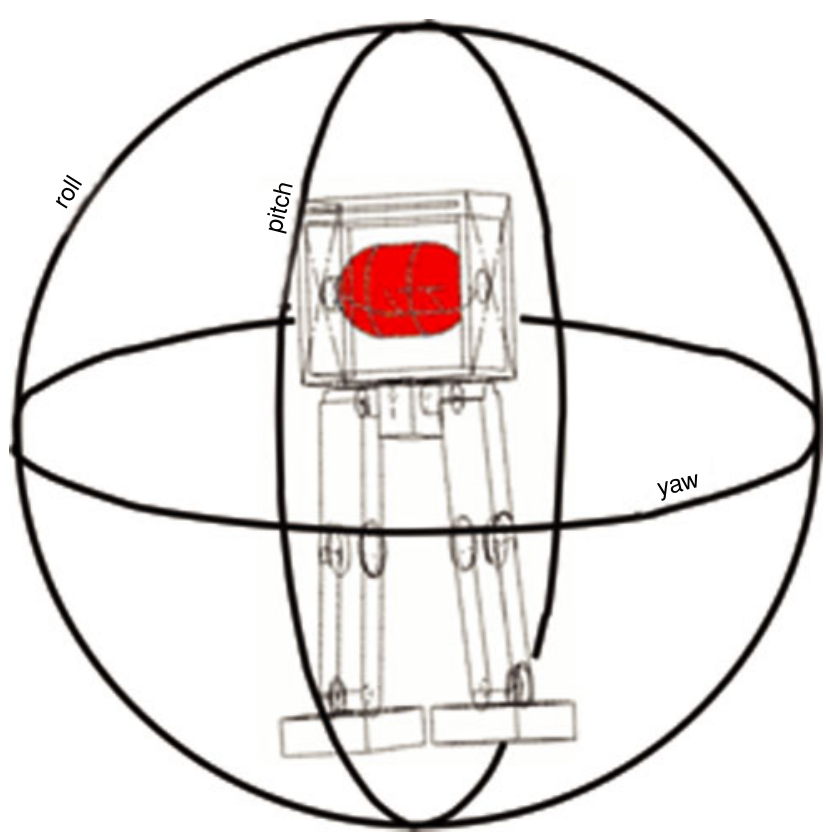

Figure 4 Design of a (hypothetical) robot. The simulated robot has 3 degrees of freedom in each leg plus the degree of freedom due to the rotor.

The design outlined here differs from previous approaches in that it uses gyros and/or reaction wheels in a way such that the axis of the gyro is parallel to the hip, thus allowing the robot's attitude to be controlled. The hip is allowed to move rhythmically forward and backward during locomotion.

\section{ROTOR-BRAKE SYSTEM FOR RAPID MOVEMENTS}

Theoretically, the gyro/reaction wheel can also be used for rapidly changing the attitude of the robot, and thus creating rapid movements like standing up from a prone position on the ground. The above-mentioned design constraints seem to make such an approach infeasible. One possible solution however is to build a rotor which can be stopped by a mechanical brake (Figure 6). In this way, the deceleration of the brake can produce high torques. Theoretical considerations show that the robot has to meet the following two requirements in order to stand up:
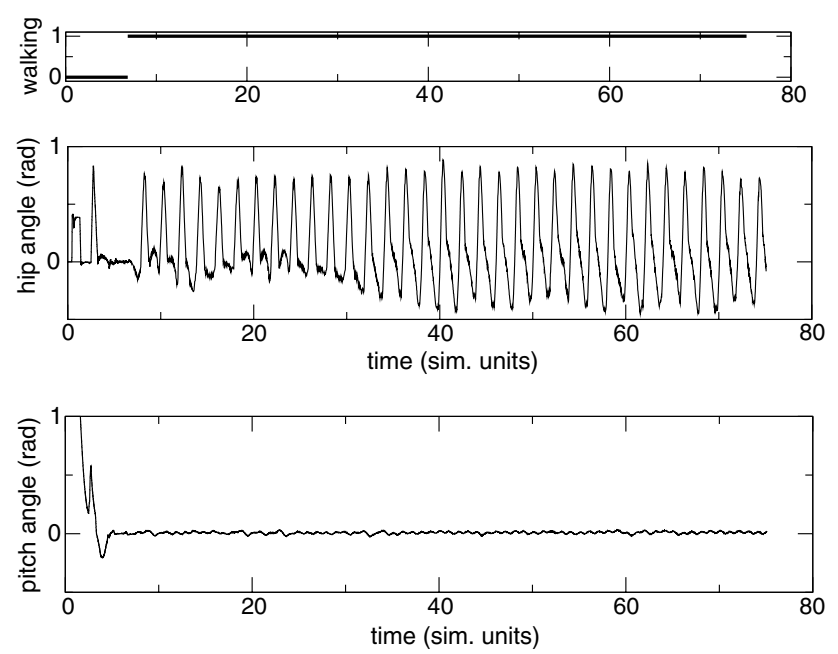

Figure 6 Walking pattern of the robot. Motion patterns of walking in the actuated biped robot. Top: the state of the walking behaviour. Middle: motion pattern of the left hip. The motion pattern is not entirely regular. Below: the state of the pitch value $(\alpha)$. A small oscillation can be seen.

1. The initial moment of a torque has to overcome the gravity. Simple theoretical considerations show that this means

$M>g \cdot m \cdot r$,

where $M$ is the moment of a torque produced by the brake, $m$ is the point of mass of the robot, $r$ is the leg length and $g$ is the gravitational constant $\left(9.81 \mathrm{~m} / \mathrm{s}^{2}\right)$. It is assumed that the mass of the robot is concentrated at one point at the end of the legs of the robot.

2. The second condition is that the angular momentum that sums up over the braking time and is then transferred to the robot has to be sufficient enough to bring the robot up. In the following, we assume that the time that the rotor requires to be stopped is significantly shorter than the time the robot requires to stand up. This means that the complete momentum from the rotor is transferred to the robot. The initial momentum of the rotor can be calculated. On the other hand, the kinetic energy has to be sufficient to bring the robot to the vertical position. After a very hard and short braking, almost the complete angular momentum $I$ of the rotor should be transferred to the robot's body, and thus $I \approx \alpha \cdot m \cdot r$.

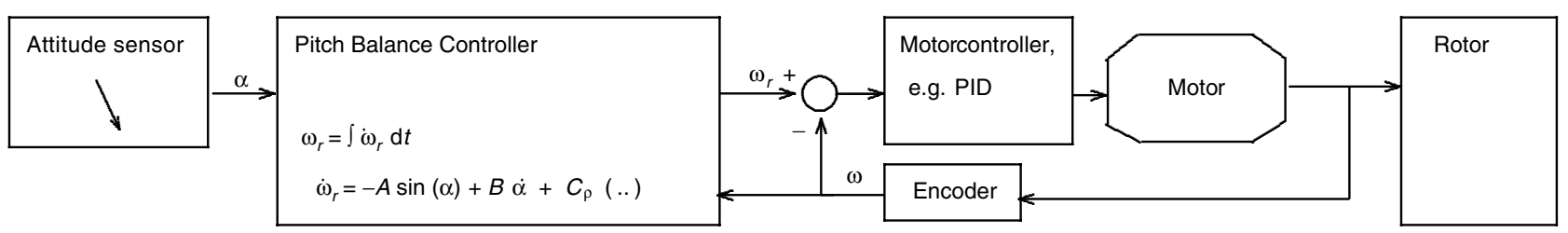

Figure 5 Scheme for the pitch balance controller: The controller uses information about the pitch angle of the robot and the encoder values from the rotor as input, giving $\alpha$ as the output (e.g., gyroscope and gravity sensor combination). The parameters $A, B$ and $C$ have to be adapted to the properties of the robot. PID indicates proportional-integral-derivative controller. 


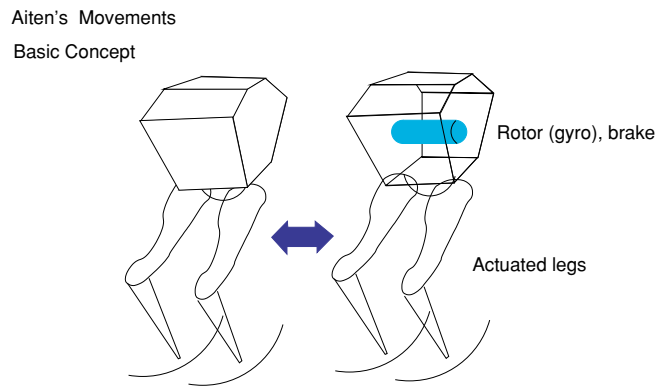

A. Balancing and standing

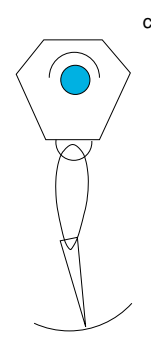

control with motor

B. Standing up (from laying on backside)
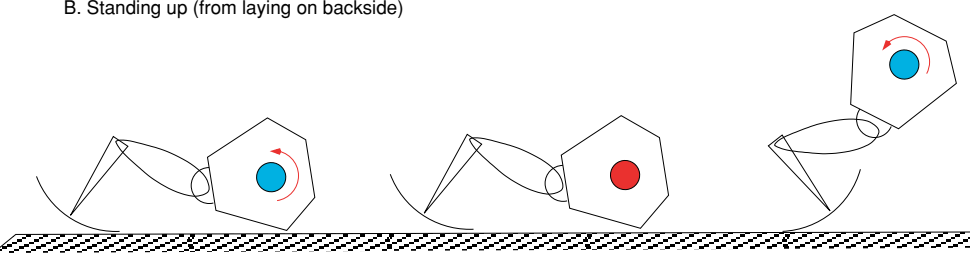

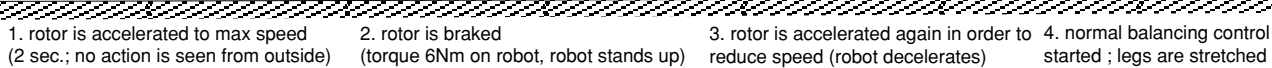

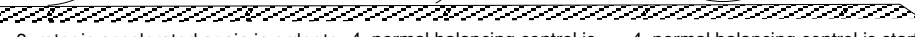

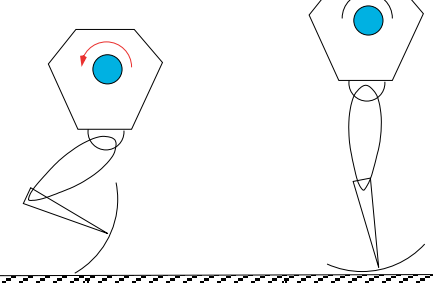

4. normal balancing control is started

C. Standing up (from laying on frontside)

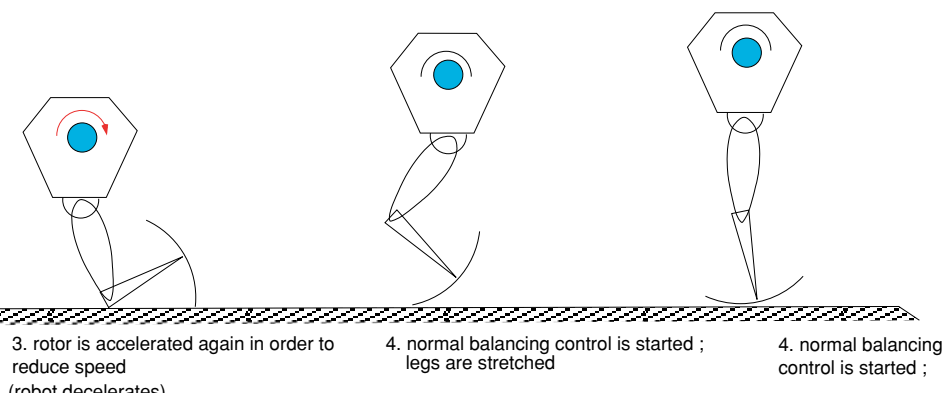

1. rotor is accelerated to max speed

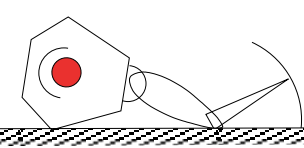

reduce speed

(torque $6 \mathrm{Nm}$ on robot, robot stands up)

(robot decelerates)

D. Walking
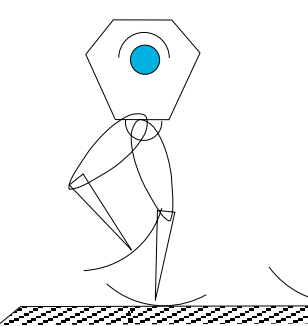
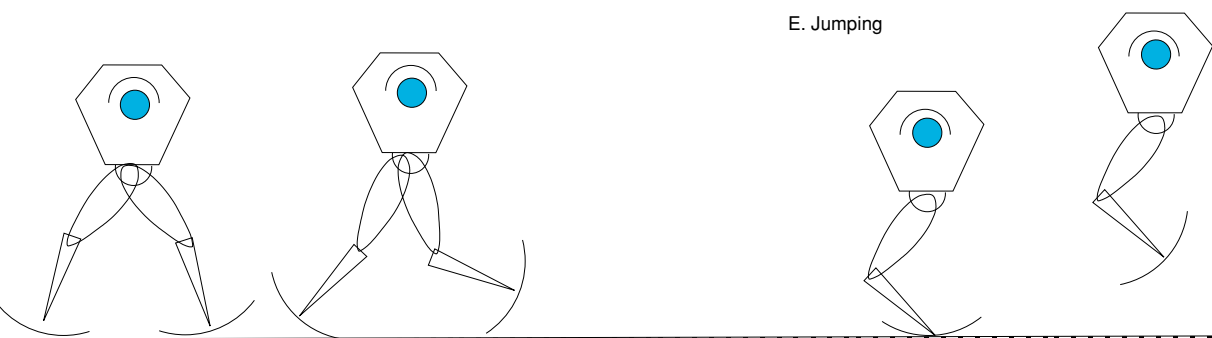

Dynamic Walking

F. Somersault
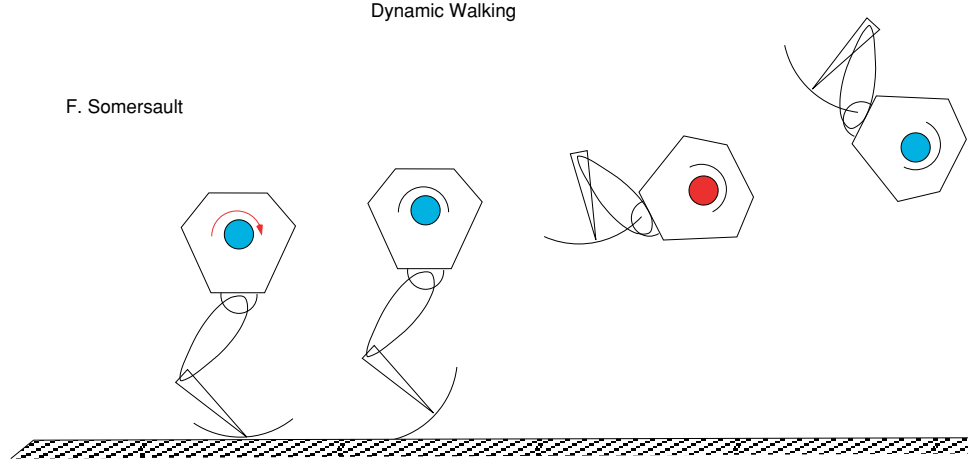

Rotor is accelerated to full speed

robot jumps like in $\mathrm{E}$

brakes....

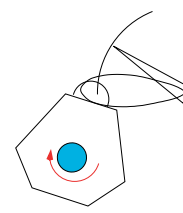

Jumping (done by toe striking cam mechanism)
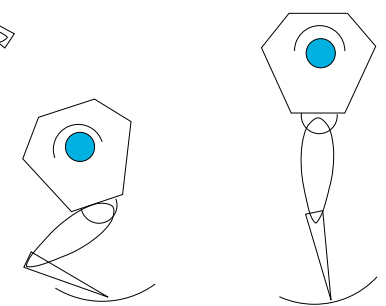

during flight rotor is accelerated

standing up catches the remaining accelerated energy

Figure 7 Possible rapid movements suggested for the NEDO artistic biped project. The figure displays possible movements of robot that uses a rotor-brake system. The movements $B$ and $F$ have been actually realised in a prototype robot at the Aichi World Expo. 
From these equations, easily the conditions for standing up, and with some modifications for other movements, can be obtained. Figure 7 shows some examples for rapid movements, of which two have been realised in a real robot prototype (NEDO 2005) in a biped robot that had relatively short kneeless legs and an additional cam/spring device for jumping.

\section{DISCUSSION}

We present here an overview of several approaches that relate to the concept of stabilising a biped robot by a gyro. The main purpose is to summarise the works and projects that relate to this idea and to give a kind of general proof of the concept. The work is still in progress, and the presented approaches rely mainly on simulations.

Our initial intention was to provide a continuous parameter that can interpolate between a $2 \mathrm{D}$ biped walker approach and a 3D biped. This can again be useful to support reinforcement and genetic control approaches to control and stabilise walking with a parameter that continuously tunes the complexity of the task (in this case, by using a different speed of the rotor). The simulation of the kneeless walker provides a perfect example of such an approach. In principle, any 2D walker can be embedded into a $3 \mathrm{D}$ world and stabilised by attaching a gyro.

The rotor-brake combinations that use the reaction wheel effects in order to produce rapid movements may be more of technical interest. This can be useful for entertainment. In particular, in combination with the gyro effect sequences of seemingly 'magic' movements are possible. This was also the conceptual idea behind the artistic biped approach 'Jumping Joe' at the Aichi World Expo near Nagoya, where a robot using this principle was presented. In addition, the principle does not require direct interaction with the environment, and can thus be interesting for other types of applications.

\section{ACKNOWLEDGMENTS}

N.M.M. thanks F. Farkas for this friendly support. The work was financially supported by Handai FRC and a KAKEN Wakate project.

\section{REFERENCES}

Collins SH, Wisse M, Ruina CA. 2001. A three-dimensional passive-dynamic walking robot with two knees and legs. Int $\mathcal{F}$ Rob Res, 20(7), 607-15.

Ferreira E, Tsai S, Paredis C, et al. 2000. Control of the Gyrover: a single-wheel gyroscopically stabilized robot. Adv Rob, 14(6), 459-75.

Garcia M, Ruina CA, Coleman MJ. 1998. The simplest walking model: stability, complexity, and scaling. ASME $\mathcal{f}$ Biomech Eng, 120(2), 281-8.

Hass J, Mayer NM, Herrmann JM. 2004. Dynamics and control of a 2-D passive dynamic walker, DY 46.35, E-Verhandlungen. In Proceedings of the DPG-Fruehjahrstagung, 8-12 March 2004, Regensburg.

Hirakoso H. 1996. Experimental research of the man-type biped locomotion robot with gyro-stabilizer (3rd Report). In Robotics and Mechatronics Conference (ROBOMEC 1996), 20-21 June 1996, vol A, p. 552-5.

Kuo AD. 1999. Stabilizing the lateral motion in passive dynamic walking. Int $\mathcal{7}$ Rob Res, 18(9), 917-30.

Mayer NM, F-Nassiraei AA, Farkas F, et al. 2004a. Stabilization of dynamic walking by using physical tricks. In Proceedings of the International Conference on Climbing and Walking Robots (CLAWAR, 2004), 22-24 September 2004, Madrid.

Mayer NM, Herrmann JM, F-Nassiraei AA, et al. 2004b. An asymmetric 2-D passive dynamic walker. In Proceedings of the AROB, 28-30 January 2004, Beppu, Oita, Japan.

McGeer T. 1990. Passive walking with knees. In Proceedings of the IEEE International Conference on Robotics and Automation, 13-18 May 1990, Los Alamitos, CA, p. 1640 5.

McGeer T. 1993. Passive dynamic walking. Int 7 Rob Res, 9(2), $62-82$.

NEDO. 2005. Robot project (CD-Rom, in Japanese). In Expo 25 March-25 September 2005, Aichi, Japan.

Schwab L, Wisse M. 2001. Basin of attraction of the simplest walking model. In ASME 2001 Design Engineering Technical Conferences, 9-12 September 2001, DETG 2001/VIB 21363.

Sugihara T, Inoue H. 2002. Real time humanoid motion generation through ZMP manipulation based on pendulum control. In IEEE International Conference on Robotics and Automation, ICRA 2002, 11-15 May 2002, p. 1404-9. 

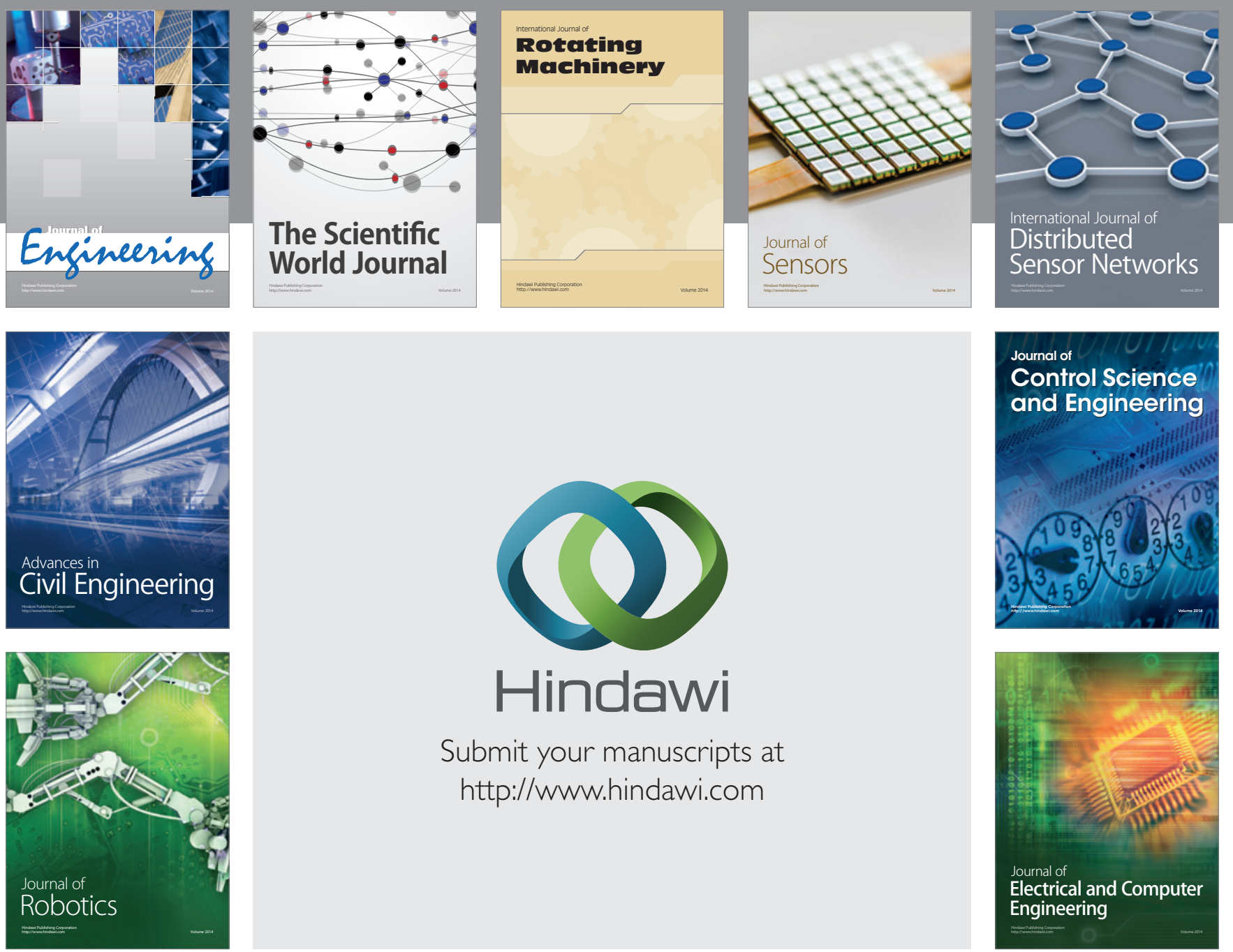

Submit your manuscripts at

http://www.hindawi.com
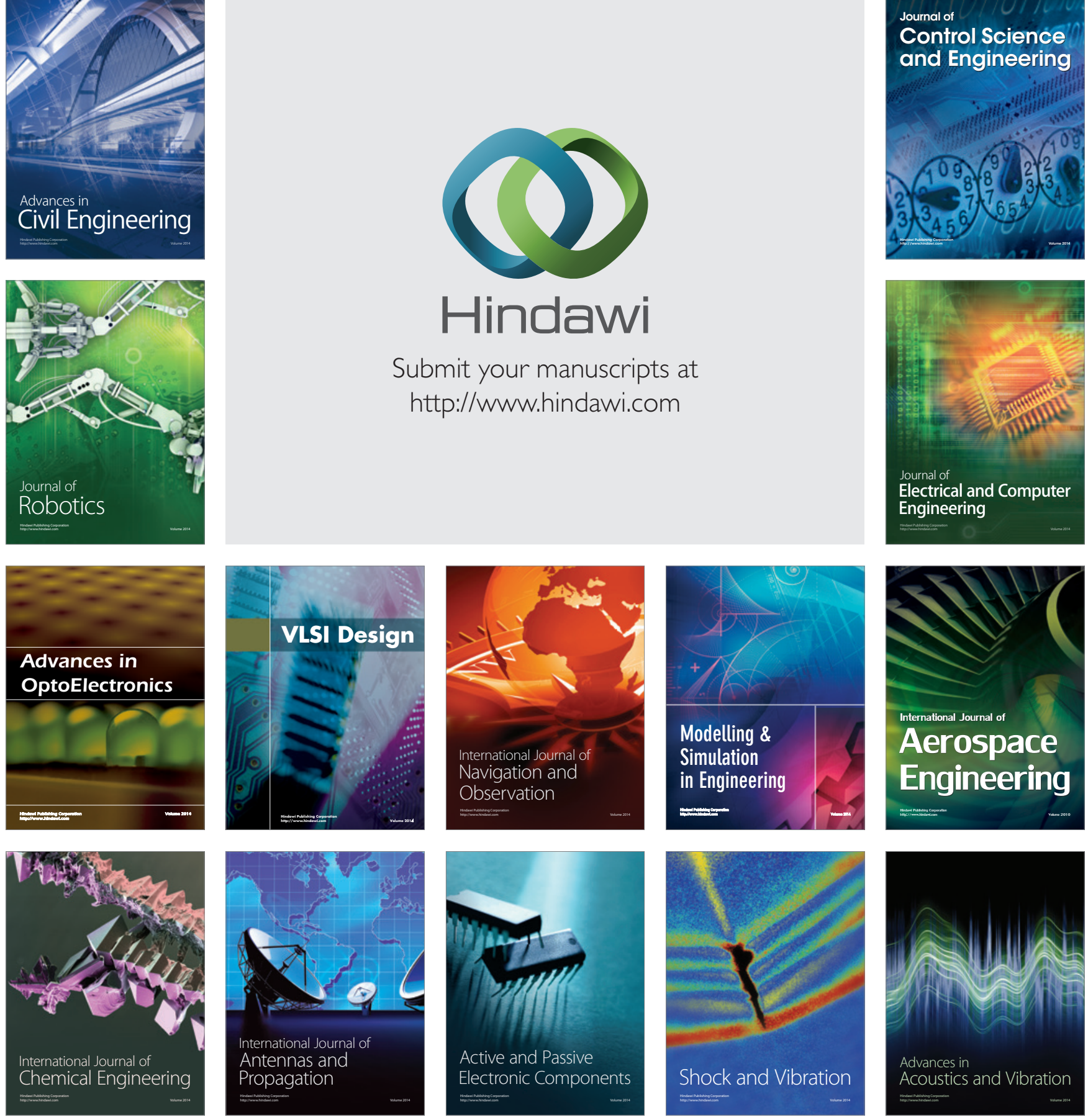